\title{
Improving bounds on outage probability in correlated Rayleigh fading environment
}

\author{
ARVIND DHAKA ${ }^{1,2}$, SIDDHARTHA CHAUHAN $^{1}, \operatorname{VIDHYACHARAN~BHASKAR~}^{3,4,5, *}$ and \\ AMITA NANDAL ${ }^{2,4}$ \\ ${ }^{1}$ Computer Science and Engineering Department, National Institute of Technology, Hamirpur, Hamirpur, India \\ ${ }^{2}$ Department of Computer and Communication Engineering, Manipal University Jaipur, Jaipur, India \\ ${ }^{3}$ Department of Electrical and Computer Engineering, San Francisco State University, 1600 Holloway Avenue, \\ San Francisco, CA 94132, USA \\ ${ }^{4}$ Department of Computer Science, San Francisco State University, 1600 Holloway Avenue, San Francisco, \\ CA 94132, USA \\ ${ }^{5}$ Department of Electrical Engineering, Northwestern Polytechnic University, 47671 Westinghouse Drive, \\ Fremont, CA 94539, USA \\ e-mail: arvind.neomatrix@gmail.com; siddharthachauhan1@gmail.com; vcharan@gmail.com; \\ amita_nandal@yahoo.com
}

MS received 17 March 2018; revised 27 March 2019; accepted 28 March 2019; published online 9 May 2019

\begin{abstract}
In this letter, we propose a limiting criterion to improve the bounds on outage probability for $n$ correlated Rayleigh fading channel. We use a threshold-based limiting distribution to yield explicit bounds on probability. This work takes into consideration the effect of correlated noise signal to the receiver, which is a generalization based on the power and noise correlation vectors. The approach proposed in this paper is different from many other existing bounds and it provides strong information to improve the results for the bounds on the outage probability.
\end{abstract}

Keywords. Correlated noise; Rayleigh fading; marginal probability; normalized power; outage probability; signal to noise ratio.

\section{Introduction}

The noise models in Rayleigh fading channels have been extensively studied over the years. The wireless channels undergo different types of fading due to noise and interferences [1-5]. Therefore, the effect of correlated noise in wireless channels is gaining attention of researchers. It is quite challenging to derive outage probability when channel is correlated. In this letter, we prove that the bounds on probability results converge explicitly using the proposed approach. We improve the bounds on outage probability using threshold-based limiting distribution. We have also analysed the proposed approach by taking into consideration the correlation among the signals, which considerably affects the outage analysis.

We have also considered the effect of correlation of noise signals in the Rayleigh fading environment. We choose the limiting criteria for threshold such that the correlation among fading signals has negligible effect on the outage probability, which improves the bound results.

*For correspondence
There is an improvement in the bounds, which gives a high value of probability. The closed-form expressions for outage probability explicitly construct the Rayleigh fading signals at receiver with sharply converging bounds, which is the main advantage of this work.

\section{System model}

We can generate $n$-correlated Rayleigh fading envelopes from $n$-dimensional independent and identically distributed (i.i.d.) complex Gaussian distribution [6] such that the Gaussian vector can be written as $V=\left[V_{1} V_{2} \cdot \cdot V_{n}\right]$, where $V_{1}=\left[a_{1}, a_{2}, \ldots, a_{n}\right], \quad V_{2}=\left[b_{1}, b_{2}, \ldots, b_{n}\right], \quad \ldots \quad$ and $V_{n}=\left[n_{1}, n_{2}, \ldots, n_{n}\right]$. Here, $a, b, \ldots, n$ are complex Gaussian random variables with zero mean and variance as $\sigma_{a}^{2}, \sigma_{b}^{2}, \ldots, \sigma_{n}^{2}$, respectively [7]. The vector $X=$ $\left[X_{a}, X_{b}, \ldots, X_{n}\right]$ is the overall magnitude vector for correlated Rayleigh-distributed random variables such that $X_{a}=\frac{X_{a a}+X_{a b}+\cdots+X_{a n}}{n}, X_{b}=\frac{X_{b b}+X_{b c}+\cdots+X_{b n}}{n-1}, \ldots, X_{n}=X_{n n}$. Let us consider that at receiver output the vector $X$ can be represented as $X=V \mathbb{F}+\mathbb{m}$. Generally, $X$ is the square root 
of sum of amplitude squares of $n$ i.i.d. complex Gaussian random variables with zero mean and $\frac{\sigma^{2}}{2}$ variance $[6,7]$. Here, $\mathbb{F}$ is the fading amplitude vector that is calculated using the correlation among different signals such that $\mathbb{F}=\left[\begin{array}{llll}\mathbb{F}_{a} & \mathbb{F}_{b} & \ldots & \mathbb{F}_{n}\end{array}\right]^{T}$ and $[.]^{T}$ represents transpose vector. The fading amplitude is affected due to noise correlation and it is dependent on average and normalized power [8]. We can represent $\mathbb{F}$ in terms of average received power $\left(P_{\text {avg }}\right)$ and power-normalized fading amplitude $\left(P_{\text {norm }}\right)$ as

$\mathbb{F}=$

$$
\left[\sqrt{P_{\text {avga }}} \cdot P_{\text {norm }}, \quad \sqrt{P_{a v g_{b}}} \cdot P_{\text {norm }}, \quad \cdots \quad \sqrt{P_{a v g_{n}}} \cdot P_{n o r m}\right]^{T} .
$$

We have used average and normalized power to describe instantaneous signal to noise ratio (SNR) later in this paper. The correlated noise matrix 0 is described $\operatorname{as} \mathbb{a}=\left[\begin{array}{ccccc}\mathbb{a}_{a a} & \mathbb{a}_{a b} & \mathbb{a}_{a c} & . . & \mathbb{a}_{a n} \\ 0 & \mathbb{a}_{b b} & \mathbb{a}_{b c} & . . & \mathbb{a}_{b n} \\ . . & . . & . . & . . & . . \\ . . & . . & . . & . . & . . \\ 0 & 0 & 0 & . . & \mathbb{a}_{n n}\end{array}\right]$. Here the noise correlation coefficient between any two variables, say $a$ and $b$, is represented as $\mathbb{a}_{a b}$ such that $\mathbb{a}_{a b}=\frac{\operatorname{Cov}\left(\left[\mathbb{m}_{1}\right]^{2},\left[\mathfrak{m}_{2}\right]^{2}\right)}{\sqrt{\operatorname{Var}\left(\left[\mathrm{m}_{1}\right]^{2}\right) \operatorname{Var}\left(\left[\mathrm{m}_{2}\right]^{2}\right)}}$. The distribution of these $n$-correlated Rayleigh-distributed random variables is computed using the procedure given in [9] as

$$
\begin{aligned}
f(X)= & \frac{2 X^{m}}{\pi|k|^{T}(1-|P|)\left(\sigma_{a} \cdot \sigma_{b} \ldots \sigma_{n}\right)^{m+1}} \cdot \mathrm{e}^{\left(\frac{2 \sqrt{|P|} X}{(1-|P|)\left(\sigma_{a} \cdot \sigma_{b} \ldots \sigma_{n}\right)}\right)} \\
& \cdot J_{m-1}\left(\frac{2 X}{\left(\sigma_{a} \cdot \sigma_{b} \ldots \sigma_{n}\right)}\right) .
\end{aligned}
$$

Here, $J_{m-1}(\cdot)$ is $(m-1)$-order Bessel function, $P$ is power correlation matrix and $k$ is correlation matrix, which is elaborated in [10]. The convergence of bounds is illustrated using correlation dependence ratio, i.e. $\frac{1}{|k|}[10]$. Therefore, our correlation model is a generalization based on the power and noise correlation vectors. Now, we can calculate the CDF of $f(X)$ by integrating $f(X)$ from 0 to $\infty$ w.r.t. $X$, i.e. $F(X)=\int_{0}^{\infty} f(X) d X$. After simplification and replacing the modified Bessel function by an infinite series [10, Eq. 9.6.10], we obtain

$$
\begin{aligned}
& F(X)=2^{n}|k|^{T} \exp \left[-\left(X_{a}^{2} P_{a}^{2}+X_{b}^{2} P_{b}^{2}+\cdots+X_{n}^{2} P_{n}^{2}\right)\right] \\
& \cdot \sum_{\{A, B, \ldots, N\}=0}^{\infty} \frac{\left|P_{a b}\right|^{2 A+m}|P|^{2 B+m} \ldots\left|P_{(n-1) n}\right|^{2(N-1)+m}}{A ! B ! \ldots N !(A+m) !(B+m) ! \ldots(N+m) !} \\
& \cdot \frac{\left|\psi_{a n}\right|^{2 N+m}}{N !(N+m) !} \cdot X_{a}^{2(B+C+D+\ldots N+m)+1} X_{b}^{2(A+C+D+\ldots N+m)+1} \\
& \ldots X_{n}^{2(A+B+C+\ldots(N-1)+m)+1} .
\end{aligned}
$$

Here, $A, B, \ldots, N \rightarrow 1,2, \ldots, n$. Now, Eq. (2) is used to evaluate outage probability.

\section{Upper and lower bounds on outage probability}

Outage probability is defined as the probability that output instantaneous SNR falls below a certain given threshold level [11]. In this letter, we have set the threshold levels within the limits of $\ell$ and $u$ as lower and upper threshold levels, respectively. These threshold levels have been used to evaluate and analyse the outage probability using limiting distribution $[12,13]$ for three cases, i.e., $\ell<\sum_{i=1}^{n} \frac{\bar{\xi}_{n} X_{n}^{2}}{E\left(X_{n}^{2}\right)}<u$, $0<\sum_{i=1}^{n} \frac{\bar{\xi}_{n} X_{n}^{2}}{E\left(X_{n}^{2}\right)}<\ell$ and $u<\sum_{i=1}^{n} \frac{\bar{\xi}_{n} X_{n}^{2}}{E\left(X_{n}^{2}\right)}<\infty$. We consider $X$ as $n$-correlated Rayleigh-distributed random variable. Let us consider $\xi_{n}$ as the instantaneous SNR of $n^{\text {th }}$ branch such that $\xi_{n}=P_{a v g_{n}} \cdot P_{n o r m_{n}}^{2}=\frac{\bar{\xi}_{n} X_{n}^{2}}{E\left(X_{n}^{2}\right)}$. Here, $\bar{\xi}_{n}$ is the average SNR at the $n^{\text {th }}$ branch and the expectation is given by $E($.$) . The$ average SNR is $\bar{\xi}_{n}=\frac{E_{t r}}{m} E\left(X_{n}^{2}\right)$. Here, $E_{t r}$ is the energy of the transmitted signal and $\frac{m}{2}$ is the power spectral density (PSD) of additive white Gaussian noise (AWGN) noise at each branch [14, 15]. We compare and select SNR based on thresholds such that overall outage probability is defined as

$$
\begin{aligned}
& \mathbb{P}\left(\sum_{i=1}^{n} \frac{\bar{\xi}_{n} X_{n}^{2}}{E\left(X_{n}^{2}\right)}\right)=\mathbb{P}\left(0<\sum_{i=1}^{n} \frac{\bar{\xi}_{n} X_{n}^{2}}{E\left(X_{n}^{2}\right)}<\ell\right) \\
& \quad+\mathbb{P}\left(u<\sum_{i=1}^{n} \frac{\bar{\xi}_{n} X_{n}^{2}}{E\left(X_{n}^{2}\right)}<\ell\right)+\mathbb{P}\left(u<\sum_{i=1}^{n} \frac{\bar{\xi}_{n} X_{n}^{2}}{E\left(X_{n}^{2}\right)}<\infty\right) .
\end{aligned}
$$

The marginal outage probability $(\mathbb{P}()$.$) [9] between upper$ $(u)$ and lower $(\ell)$ threshold level is written as

$$
\begin{aligned}
& \mathbb{P}\left(\ell<\sum_{i=1}^{n} \frac{\bar{\xi}_{n} X_{n}^{2}}{E\left(X_{n}^{2}\right)}<u\right) \\
& =\sum_{i=1}^{n} \frac{(u+\ell) e^{-\frac{(u+\ell)^{2} X_{i}^{2}}{2}}}{\sqrt{2 \pi}} \cdot \int_{\ell}^{u} F(X) d X .
\end{aligned}
$$

When $0<\sum_{i=1}^{n} \frac{\bar{\xi}_{n} X_{n}^{2}}{E\left(X_{n}^{2}\right)}<\ell$ then there exists a strict lower bound probability as

$$
\begin{gathered}
\mathbb{P}\left(0<\sum_{i=1}^{n} \frac{\bar{\xi}_{n} X_{n}^{2}}{E\left(X_{n}^{2}\right)}<\ell\right) \geq \sum_{i=1}^{n} \frac{e^{-\frac{X_{i}^{2}}{2}}}{\sqrt{2 \pi}} \\
\cdot\left[\frac{(1-\ell)\left(\ell-\sum_{j=1}^{n} X_{j}^{2}\right)}{\sqrt{1-\sum_{j=1}^{n} X_{j}^{2}}}\right] \cdot \int_{0}^{\ell} F(X) d X .
\end{gathered}
$$


When $u<\sum_{i=1}^{n} \frac{\bar{\xi}_{n} X_{n}^{2}}{E\left(X_{n}^{2}\right)}<\infty$ then there exists a strict upper bound probability as

$$
\begin{gathered}
\mathbb{P}\left(u<\sum_{i=1}^{n} \frac{\bar{\xi}_{n} X_{n}^{2}}{E\left(X_{n}^{2}\right)}<\infty\right) \geq \sum_{i=1}^{n} \frac{e^{-\frac{X_{i}^{2}}{2}}}{\sqrt{2 \pi}} \\
\cdot\left[\frac{(u-1)\left(\sum_{j=1}^{n} X_{j}^{2}-u\right)}{\sqrt{1-\sum_{j=1}^{n} X_{j}^{2}}}\right] \cdot \int_{u}^{\infty} F(X) d X .
\end{gathered}
$$

Now, we set the limit on the selection of $\ell$ and $u$ with the help of limiting distribution parameters [12,13] to improve the product term of Eqs. (4)-(6). For the lower bound, we select the limit of $\ell$ between $\alpha_{\text {low }}$ and $\beta_{\text {low }}$ such that $\alpha_{\text {low }}=\frac{\ell}{\sqrt{2 \pi}}$ and $\beta_{\text {low }}=\frac{\ell}{\sqrt{1-\sum_{j=1}^{n} X_{j}^{2}}}$. For the upper bound, we select the limit of $u$ between $\alpha_{u p}$ and $\beta_{u p}$ such that $\alpha_{u p}=\frac{u}{\sqrt{2 \pi}}$ and $\beta_{u p}=$ $\frac{u}{\sqrt{1-\sum_{j=1}^{n} X_{j}^{2}}}$. Thus, for suitable lower bound $\alpha_{\text {low }} X_{i}<\frac{\partial \ell}{\partial \beta_{\text {low }}}$ and for suitable upper bound $\alpha_{u p} X_{i}<\frac{\partial u}{\partial \beta_{u p}}$. The overall values are $\alpha=\alpha_{\text {up }}-\alpha_{\text {low }}$ and $\beta=\beta_{\text {up }}-\beta_{\text {low }}$. This situation allows some flexibility to the probability level of upper and lower bounds such that these probabilities are never too small; $\alpha$ and $\beta$ control the threshold criterion for outage analysis in such a way that probability results are improved. Using the limiting distribution parameters $\left(\alpha_{\text {low }}\right.$ and $\left.\beta_{\text {low }}\right)$ we can rewrite Eqs. (4), (5) and (6) as Eqs. (7), (8) and (9), respectively such that

$$
\begin{gathered}
\mathbb{P}\left(\ell<\sum_{i=1}^{n}\left|X_{i}^{2}\right|<u\right)=\sum_{i=1}^{n} \frac{(\alpha+\beta) e^{-\frac{(\alpha+\beta)^{2} X_{i}^{2}}{2 \pi}} \cdot \int_{\ell}^{u} F(X) d X}{\sqrt{2 \pi}} \\
\mathbb{P}\left(0<\sum_{i=1}^{n}\left|X_{i}^{2}\right|<\ell\right) \geq \sum_{i=1}^{n} \frac{e^{-\frac{X_{i}^{2}}{2}}}{\sqrt{2 \pi}} \\
\cdot\left[\frac{\left(1-\alpha_{\text {low }}-\beta_{\text {low }}\right)\left(\alpha_{\text {low }}+\beta_{\text {low }}-\sum_{j=1}^{n} X_{j}^{2}\right)}{\sqrt{1-\sum_{j=1}^{n} X_{j}^{2}}}\right] \\
\left.\cdot \int_{0}^{\ell} F(X) d X, \quad\right] \\
\mathbb{P}\left(u<\sum_{i=1}^{n}\left|X_{i}^{2}\right|<\infty\right) \geq \sum_{i=1}^{n} \frac{e^{-\frac{x_{i}^{2}}{2}}}{\sqrt{2 \pi}} \\
\quad\left[\frac{\left(\alpha_{u p}+\beta_{u p}-1\right)\left(-\left(\alpha_{u p}+\beta_{u p}-\sum_{j=1}^{n} X_{j}^{2}\right)\right)}{\sqrt{1-\sum_{j=1}^{n} X_{j}^{2}}}\right] . \\
\qquad \int_{u}^{\infty} F(X) d X .
\end{gathered}
$$

It is observed from Eqs. (7)-(9) that due to limiting distribution parameters, the product term is now sufficiently large, which results in sharper bound.

\section{Numerical results and discussion}

We illustrate the performance in terms of bounds obtained by plotting outage probability w.r.t. threshold. For the computation of outage probability we have evaluated the probability distributions using MATLAB software. We have observed that the probability function converges towards neighbourhood of $\ell=20 \mathrm{~dB}$ and $u=0 \mathrm{~dB}$. We have shown outage probability bounds for theoretical and simulated values in figure 1. By carefully looking at the results we observe that bounds attain a critical value, which delivers exact inference so that the plots become sharper in the sense of achieving better level of probability results. Figure 2 shows the outage probability for different values of threshold for correlated Rayleigh fading channel with threshold level 5, 15 and $20 \mathrm{~dB}$. It is observed from figure 2 that the outage probabilities are partially merged for different values of outage threshold. It is observed that both upper and lower bounds converge to the exact outage probability when the value of SNR is above $10 \mathrm{~dB}$. When

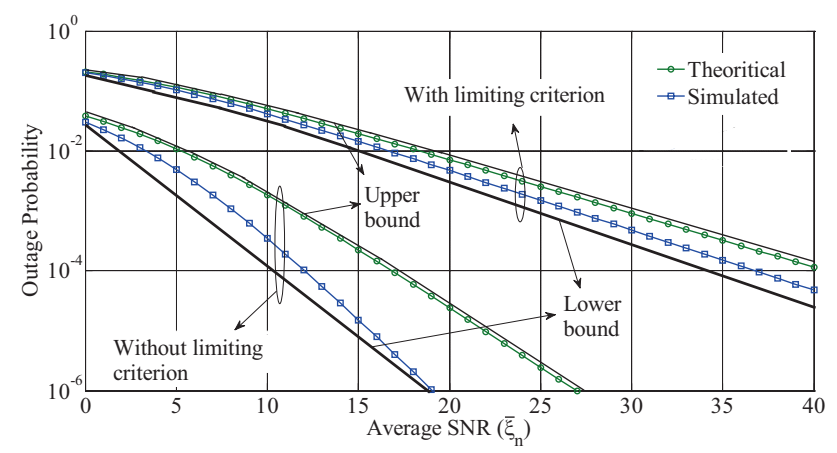

Figure 1. Outage probability versus average SNR with theoretical and simulated results.

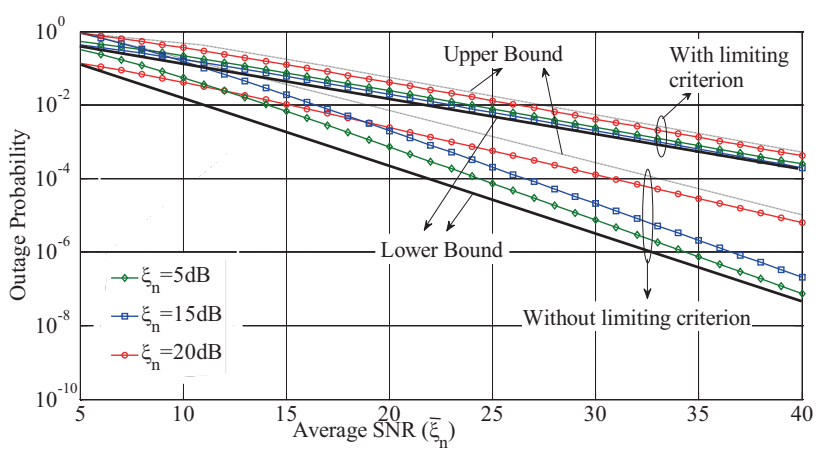

Figure 2. Outage probability versus average SNR for different threshold levels. 
the value of SNR is above $20 \mathrm{~dB}$ then outage probability is below $10^{-10}$, which signifies that larger computational time is required to simulate the evaluated outage expressions. According to figure 2 the correlation dependence ratio is observed such that when SNR is low then this ratio is also small and when SNR is high then this ratio is large. The outage probability bounds become tighter using limiting criterion as shown in figure 2. It is observed that the gap between outage probability values is less when limiting criterion is used. This means that bounds converge faster when the gap is small. Therefore, it is observed that bounds are sharper with the help of limiting distribution criterion.

\section{Conclusion}

This letter covers strong approximation of lower and upper bounds on outage probability for $n$-correlated Rayleigh fading channel. This paper provides step by step implementation of the proposed method. We present the closedform expression for bound estimates. We have also provided numerical simulation results for outage probability under different threshold conditions. It is concluded that the estimated bounds tend to be sharper using proposed limiting threshold criterion.

\section{References}

[1] Blumenson L E and Miller K S 1963 Properties of generalized Rayleigh distributions. Ann. Math. Stat. 34: 903-910

[2] Akuon O P and X H 2016 Optimal error analysis of receive diversity schemes on arbitrarily correlated Rayleigh fading channels. IET Commun. 10: 854-861

[3] Hong L and Armada A G 2011 Bit error rate performance of MIMO MMSE receivers in correlated Rayleigh flat-fading channels. IEEE Trans. Veh. Technol. 60: 313-317
[4] Dhaka A, Chauhan S and Bhaskar V 2018 Analysis and simulation of second-order statistics with modified characteristic function parameters in a multipath fading environment. Wirel. Pers. Commun. 100: 851-862

[5] Dhaka A, Chauhan S and Bhaskar V 2018 Effect of matrix partitioning on second order statistics of fading channels. Wirel. Pers. Commun. 100: 863-875

[6] Proakis J G 1995 Digital communications, 3rd ed. New York: McGraw-Hill

[7] Dhaka A, Chauhan S, Bhaskar V and Marina N 2018 A statistical approach using phase variance for analyzing correlated multipath fading environment. IET Commun. 12: 948-955

[8] Lee M and Oh S K 2013 Joint power allocation for coordinated two-point diversity transmission under a coordinated power constraint in Rayleigh fading channels. IEEE Commun. Lett. 17: 1104-1107

[9] Miller K S 1975 Multivariate distributions. New York: RE Krieger Pub. Co.

[10] Abramowitz M and Stegun I A 1972 Handbook of mathematical functions with formulas, graphs, and mathematical tables. Washington, D.C., USA: United States Department of Commerce, National Institute of Standards and Technology (NBS)

[11] Annavajjala R and Milstein L B 2005 Performance analysis of linear diversity-combining schemes on Rayleigh fading channels with binary signaling and Gaussian weighting errors. IEEE Trans. Wirel. Commun. 4: 2267-2278

[12] Shao Q M 1996 Bounds and estimators of a basic constant in extreme value theory of Gaussian processes. Stat. Sin. 6: $245-257$

[13] Leadbetter M R, Lindgren G and Rootzen H 1983 Extremes and related properties of random sequences and processes. New York: Springer

[14] Subhashini J and Bhaskar V 2015 Performance analysis of Rayleigh fading channels with hybrid correlation effects with 2-branch maximal ratio combining. Wirel. Pers. Commun. 83: $673-688$

[15] Bhaskar V and Subhashini J 2014 Spectrum efficiency evaluation with diversity combining for fading and branch correlation impairments. Wirel. Pers. Commun. 79: $1089-1110$ 